\title{
VOLUMETRIC GROWTH OF THERMOELASTIC MATERIALS AND MIXTURES
}

\author{
Stephen M. Klisch and Anne Hoger \\ Department of Mechanical and Aerospace Engineering \\ University of California at San Diego
}

Mathematics and Mechanics of Solids 8:377-402, 2003

Address correspondence to:

Stephen M. Klisch, Ph.D.

Department of Mechanical Engineering

California Polytechnic State University

San Luis Obispo, CA 93407

(805) 756-1308; FAX (805) 756-1137

$\underline{\text { sklisch@calpoly.edu }}$ 


\begin{abstract}
The proteoglycan and collagen constituents of cartilage serve distinct mechanical roles. Changes to the mechanical loading conditions during cartilage growth lead to changes in the concentrations of these molecules and, consequently, the mechanical properties. The main aim of this paper is to present a theory that can describe the mechanical aspects of cartilage growth. The model for cartilage growth is based on a general thermomechanical theory for a mixture of an arbitrary number of growing elastic constituents and an inviscid fluid. Our development of a growth mixture theory is accomplished in two steps. First, the thermodynamics of growing elastic materials are considered. The resulting theory of growing thermoelastic materials is extended to continuum mixture theory. Using this general growth mixture theory, we then propose a cartilage growth model that includes two special types of internal constraints that are relevant to cartilage.
\end{abstract}

\title{
1. Introduction
}

The extracellular matrix of cartilage tissue is composed primarily of three constituents: water, collagen, and proteoglycans. The latter two constituents form a solid collagenproteoglycan matrix. These two molecular constituents appear to be predominantly responsible for the tissue's mechanical properties, and each serves a distinct functional role. The proteoglycan constituent provides the tissue with a fixed negative charge that increases the tissue's propensity to swell and to resist compressive loading [1]. The collagen network resists the swelling tendency of the proteoglycans, and provides the tissue with tensile and shear strength [2-3]. 
A key feature of cartilage growth is that cellular metabolism, which governs the growth, maintenance, and degradation of the extracellular matrix, is regulated by mechanical stimuli. In vivo models of experimental arthritis [4-5] and joint immobilization [6-7] suggest that altered mechanical loads, which change the local tissue stress, lead to changes in the composition and the mechanical properties of the extracellular matrix. Indeed, the metabolic response to mechanical stimuli has been quantified using in vitro experiments with tissue explants and cell cultures. For example, cell metabolism has been shown to depend on hydrostatic pressure [8], dynamic compressive stress [9-10], and fluid-induced shear stresses [11]. Furthermore, these in vitro experiments have quantified the cellular metabolic activity related to both proteoglycan and collagen synthesis.

Since the proteoglycan and collagen constituents of cartilage serve distinct mechanical roles, a model of cartilage growth that can predict the evolution of the tissue's mechanical properties during growth should allow these constituents to grow independently of each other. The main aim of this paper is to present such a theory for cartilage growth. The growth mixture theory developed here extends previous work on the growth of elastic materials [12-15]. In those papers, the material added during growth was assumed to have the same mechanical properties as the original material. The deformation gradient due to growth was decomposed into two parts: a growth tensor that describes the amount and orientation of mass deposition, and an elastic accommodation tensor that ensures the continuity of the growing body. Furthermore, the stress constitutive equation only depended on the elastic accommodation tensor and the residual stress. Taber and colleagues have used models based on [13] to study the stress-modulated growth of both the heart [16] and the aorta [17-18] using several growth laws. Recently, the 
theory has been further extended by defining the growth law on the current configuration of the growing material $[12,19]$; this implementation was used to model the growth of aortic tissue [19].

Continuum mixture theory has been used to model the finite deformation and flowindependent mechanical properties of both articular cartilage [20] and the intervertebral disc [21]. These models are based on modern mixture theory (e.g., see [22-26]). Krishnaswamy and Batra [27] developed a theory of mixtures that included a new statement of the Second Law of Thermodynamics. In that paper, a prescription for the partial entropy function was obtained for a homothermal quasi-static process, which was assumed to be a path-independent process. Using this approach, a series of experiments is identified that allows the measurement of the partial entropy function. Because of its direct relation to experiment, this approach is appealing for developing a physical understanding of the thermodynamics of mixtures. The approach of [27] was advocated in continuum thermodynamics by Day [28], Rivlin [29-30], and Casey and Krishnaswamy [31], and has been extended to the case of different constituent temperatures [32].

In this paper, a growth mixture theory is developed that employs both the mechanics of growing elastic materials presented in [12-15] and the thermodynamics of mixtures presented in [27]. The specific aim of this paper is to develop a thermomechanical theory for a mixture of an arbitrary number of growing elastic constituents and an inviscid fluid. The development here of a growth mixture theory is accomplished in two steps. First, the thermodynamics of growing elastic materials are considered. Then, this resulting theory of growing thermoelastic materials is extended to continuum mixture theory. 
A unifying theme in [27-32] is the assumption of a path-independent (or reversible) process that leads to a prescription for the entropy function. Here, we establish that there exist growing thermoelastic materials that cannot experience reversible growth, and a modified approach for applying the Second Law of Thermodynamics is presented. This approach is motivated by the idea that, at any time during a continuous growth process, the growth process may be stopped and experiments may be performed on excised tissue specimens to determine the thermomechanical properties (see Section 3). When the growth process is stopped, the material behaves as a thermoelastic material, for which homothermal processes are reversible. In this paper, this thermoelastic material is called the generating material. Thus, at any time during a continuous growth process, the growing thermoelastic material can be related to a single generating material. In this paper it is assumed that the thermomechanical response functions of a growing thermoelastic material are inherited from the generating material.

Section 2 summarizes previous work on thermoelastic materials [31], mixtures [27], and growing elastic materials [12]. In Section 3, a theory of growth for thermoelastic materials is proposed. This theory is extended to mixtures with growing constituents in Section 4. In Section 5, a cartilage growth model is proposed that includes two special types of internal constraints.

\section{Background}

\subsection{Thermoelastic materials}

In this section, the theory of thermoelastic materials presented by Casey and Krishnaswamy [31] is summarized in its Eulerian form. Let $B$ be a thermoelastic body with a fixed reference 
configuration $\kappa_{0}(B)$ and a configuration $\kappa(B)$ at time t. A particle of $B$ occupies positions $\mathbf{X} \in \kappa_{0}(B)$ and $\mathbf{x} \in \kappa(B)$. Let $\theta>0$ denote the absolute temperature of $B$ in $\kappa(B)$, and take $\theta_{0}$ to be the reference temperature in $\kappa_{0}(B)$. The motion and temperature history of $B$ are defined by smooth mappings

$$
\mathbf{x}=\chi(\mathbf{X}, \mathrm{t}), \quad \theta=\Theta(\mathbf{X}, \mathrm{t}) .
$$

Let $\mathbf{v}, \mathbf{F}, \mathbf{L}$, and $\mathbf{D}$ denote the velocity, deformation gradient tensor, velocity gradient tensor, and the rate of deformation tensor, respectively. It is assumed that the determinant of $\mathbf{F}$ satisfies $\mathrm{J}=\operatorname{det} \mathbf{F}>0$.

The balance equations for mass, linear momentum, angular momentum, and energy take the form

$$
\dot{\rho}+\rho \operatorname{div} \mathbf{v}=0, \quad \operatorname{div} \mathbf{T}+\rho \mathbf{b}=\rho \dot{\mathbf{v}}, \quad \mathbf{T}^{\mathrm{T}}=\mathbf{T}, \quad \dot{\rho} \dot{\varepsilon}=\rho \mathrm{r}-\operatorname{div} \mathbf{q}+\mathbf{T} \cdot \mathbf{D}
$$

on $\kappa(B)$, where $\rho$ is the mass density, $\mathbf{T}$ is the Cauchy stress tensor, $\mathbf{b}$ is the external body force, $\varepsilon$ is the internal energy, $\mathrm{r}$ is the external heat supply, and $\mathbf{q}$ is the heat flux vector.

A thermoelastic material $m$ has constitutive equations ${ }^{1}$

$$
\varepsilon=\tilde{\varepsilon}(\mathbf{F}, \theta), \quad \mathbf{T}=\tilde{\mathbf{T}}(\mathbf{F}, \theta), \quad \mathbf{q}=\tilde{\mathbf{q}}(\mathbf{F}, \theta, \mathbf{g}),
$$

where $\mathbf{g}=\operatorname{grad} \theta, \tilde{\varepsilon}\left(\mathbf{0}, \theta_{\mathrm{o}}\right)=0$, and $\tilde{\mathbf{q}}(\mathbf{F}, \theta, \mathbf{0})=\mathbf{0}$. Due to invariance requirements, $(2.3)_{1}$ may be written as

$$
\varepsilon=\hat{\varepsilon}(\mathbf{C}, \theta),
$$

where $\mathbf{C}=\mathbf{F}^{\mathrm{T}} \mathbf{F}$.

\footnotetext{
${ }^{1}$ The energy equation $(2.2)_{4}$ and the Second Law of Thermodynamics is used to obtain the result that the Cauchy stress $\mathbf{T}$ is derived from the internal energy function $\varepsilon$.
} 
In [31], the Second Law of Thermodynamics is stated in two parts. The first part is the assumption that a homothermal process (i.e., $\mathbf{g}=\mathbf{0}$ ) is path-independent; consequently, the Clausius integral derived from the energy equation for a homothermal process is integrable.

This leads to a prescription for the entropy function

$$
\eta=\hat{\eta}(\mathbf{C}, \theta),
$$

which for a homothermal process satisfies $\dot{\eta}=r / \theta$. Furthermore, it is assumed that $\eta$ depends only on $\mathbf{C}$ and $\theta$ for all processes. The Helmholtz free energy function is defined as

$$
\psi=\hat{\psi}(\mathbf{C}, \theta)=\varepsilon-\eta \theta,
$$

where $\hat{\psi}\left(\mathbf{0}, \theta_{\mathrm{O}}\right)=0$. With (2.4-2.6) and the energy equation for homothermal processes, the following constitutive equations are obtained:

$$
\mathbf{T}=2 \rho \mathbf{F} \frac{\partial \hat{\psi}}{\partial \mathbf{C}} \mathbf{F}^{\mathrm{T}}, \quad \eta=-\frac{\partial \hat{\psi}}{\partial \theta},
$$

which are valid for all processes, since none of the variables that appear in (2.7) depend on $\mathbf{g}$.

The second part of the statement of the Second Law of Thermodynamics made in [31] is the assumption of an entropy inequality that holds for all processes and which restricts the change in entropy. Here, we use the Clausius-Duhem inequality,

$$
\rho \dot{\eta}-\rho \frac{\mathrm{r}}{\theta}+\operatorname{div} \frac{\mathbf{q}}{\theta} \geq 0
$$

which leads to the heat conduction inequality:

$$
-\mathbf{q} \cdot \mathbf{g} \geq 0 .
$$


In summary, a process (in this case, a homothermal one) has been identified which is assumed to be path-independent, or reversible, ${ }^{2}$ and which leads to the identification of an entropy function.

In extending these ideas to growing materials, we must first determine if there exist growing materials which cannot experience reversible growth. In Section 3, we state and prove a theorem that identifies a necessary condition for a growth process to be reversible, and show that there exist materials that cannot experience reversible growth. Thus, an alternative statement of the Second Law of Thermodynamics is necessary. This approach is first developed for thermoelastic materials in Section 3 and extended to continuum mixture theory in Section 4.

\subsection{Mixtures of elastic materials}

In this section, the work of Krishnaswamy and Batra [27] on solid-fluid mixtures is extended to a mixture with an arbitrary number of solid elastic constituents and one inviscid fluid.

Consider a mixture $B$ of $v$ constituents: ${ }^{3} v-1$ elastic materials $C^{\alpha}(\alpha \in[1, v-1])$ and an inviscid fluid $C^{v}$. Let $\kappa_{0}(B)$ be a fixed reference configuration and $\kappa(B)$ the configuration at time t. A particle of $C^{\alpha}$ occupies positions $\mathbf{X}^{\alpha} \in \kappa_{0}(B)$ and $\mathbf{x}^{\alpha} \in \kappa(B)$. It is assumed that there exists one particle of each constituent at every point $\mathbf{x}$ in the mixture so that $\mathbf{x}=\mathbf{x}^{\alpha}$. The motion of $C^{\alpha}$ and the common temperature of $B$ are defined by smooth mappings

$$
\mathbf{x}^{\alpha}=\chi^{\alpha}\left(\mathbf{X}^{\alpha}, \mathrm{t}\right), \quad \theta=\Theta\left(\mathbf{X}^{\alpha}, t\right),
$$

where $\theta>0$ is the absolute temperature of the mixture.

The density of $C^{\alpha}$ is $\rho^{\alpha}$, and the density of the mixture is defined by

\footnotetext{
${ }^{2}$ We consider a path-independent process to be equivalent to a reversible process for a thermoelastic material.

${ }^{3}$ Superscripts when used will refer to all constituents unless noted otherwise. We closely follow the theory and notation of Craine et al. [24], who developed a mixture theory for an arbitrary number of constituents.
} 


$$
\rho=\sum_{\alpha=1}^{v} \rho^{\alpha}
$$

The velocity $\mathbf{v}^{\alpha}$, mean velocity $\mathbf{v}$, and relative velocity $\mathbf{a}^{\alpha}$ (relative to the fluid constituent $C^{v}$ ) are defined through

$$
\mathbf{v}^{\alpha}=\frac{\mathrm{d}^{\alpha}}{\mathrm{dt}} \chi^{\alpha}\left(\mathbf{X}^{\alpha}, \mathrm{t}\right), \quad \rho \mathbf{v}=\sum_{\alpha=1}^{v} \rho^{\alpha} \mathbf{v}^{\alpha}, \quad \mathbf{a}^{\alpha}=\mathbf{v}^{\alpha}-\mathbf{v}^{v}
$$

For vector functions $\mathbf{w}(\mathbf{x}, \mathrm{t})$, the material time derivatives $\mathrm{d}^{\alpha}(\cdot) / \mathrm{dt}$ following the motion of $C^{\alpha}$ and $\mathrm{d}(\cdot) / \mathrm{dt}$ following the mean mixture motion are given by

$$
\frac{\mathrm{d}^{\alpha} \mathbf{w}}{\mathrm{dt}}=\frac{\partial \mathbf{w}}{\partial \mathrm{t}}+(\operatorname{grad} \mathbf{w}) \mathbf{v}^{\alpha}, \quad \frac{\mathrm{d} \mathbf{w}}{\mathrm{dt}}=\frac{\partial \mathbf{w}}{\partial \mathrm{t}}+(\operatorname{grad} \mathbf{w}) \mathbf{v}
$$

For each $C^{\alpha}$, let $\mathbf{F}^{\alpha}, \mathbf{L}^{\alpha}, \mathbf{D}^{\alpha}$, and $\mathbf{W}^{\alpha}$ denote the deformation gradient tensor $\left(\mathbf{J}^{\alpha}=\operatorname{det} \mathbf{F}^{\alpha}>0\right)$, velocity gradient tensor, the rate of deformation tensor, and the spin tensor, respectively.

With the assumption that internal mass exchange among the constituents does not take place, the balance equations for mass, linear momentum, and angular momentum for $C^{\alpha}$ take the form

$$
\frac{\mathrm{d}^{\alpha} \rho^{\alpha}}{\mathrm{dt}}+\rho^{\alpha} \operatorname{divv}^{\alpha}=0, \quad \rho^{\alpha} \frac{\mathrm{d}^{\alpha} \mathbf{v}^{\alpha}}{\mathrm{dt}}=\operatorname{div} \mathbf{T}^{\alpha}+\pi^{\alpha}+\rho^{\alpha} \mathbf{b}^{\alpha}, \quad \mathbf{T}^{\alpha}-\mathbf{T}^{\alpha^{\mathrm{T}}}=\Lambda^{\alpha},
$$

on $\kappa(B)$, where $\mathbf{T}^{\alpha}$ is the partial Cauchy stress tensor, $\boldsymbol{\pi}^{\alpha}$ is the diffusive force, $\mathbf{b}^{\alpha}$ is the partial external body force, and $\Lambda^{\alpha}$ is the internal body couple. The balances of linear momentum and angular momentum for the mixture require that

$$
\sum_{\alpha=1}^{v} \pi^{\alpha}=\mathbf{0}, \quad \sum_{\alpha=1}^{v} \Lambda^{\alpha}=\mathbf{0}
$$

The balance of energy for $C^{\alpha}$ is 


$$
\rho^{\alpha} \frac{d^{\alpha} \varepsilon^{\alpha}}{d t}=\rho^{\alpha} r^{\alpha}-\operatorname{div} \mathbf{q}^{\alpha}+\gamma^{\alpha}+\mathbf{T}^{\alpha} \cdot \mathbf{D}^{\alpha}
$$

where $\varepsilon^{\alpha}$ is the partial internal energy, $\mathrm{r}^{\alpha}$ is the partial external heat supply, $\mathbf{q}^{\alpha}$ is the partial heat flux vector, and $\gamma^{\alpha}$ is the internal energy supply. The balance of energy for the mixture is

$$
\sum_{\alpha=1}^{v}\left(\rho^{\alpha} \frac{d^{\alpha} \varepsilon^{\alpha}}{d t}-\rho^{\alpha} r^{\alpha}+\operatorname{div} \mathbf{q}^{\alpha}+\pi^{\alpha} \cdot \mathbf{v}^{\alpha}-\mathbf{T}^{\alpha} \cdot \mathbf{L}^{\alpha}\right)=0,
$$

which can be written in the alternative form

$$
\sum_{\alpha=1}^{v}\left(\gamma^{\alpha}+\pi^{\alpha} \cdot \mathbf{v}^{\alpha}-\mathbf{T}^{\alpha} \cdot \mathbf{W}^{\alpha}\right)=0
$$

Let $\Delta$ denote the set of equilibrium state variables:

$$
\Delta=\left\{\mathbf{F}^{1}, \ldots, \mathbf{F}^{v-1}, \rho^{v}, \mathbf{G}^{1}, \ldots, \mathbf{G}^{v-1}, \operatorname{grad} \rho^{v}, \theta\right\}
$$

where $\mathbf{G}^{\alpha}=\operatorname{Grad}^{\alpha}$. Then, the internal energy, stress, and diffusive forces for each constituent may be decomposed into equilibrium and non-equilibrium terms:

$$
\begin{gathered}
\varepsilon^{\alpha}={ }_{\mathrm{o}} \varepsilon^{\alpha}(\Delta)+\mathrm{e} \varepsilon^{\alpha}\left(\Delta, \mathbf{a}^{1}, \ldots, \mathbf{a}^{v-1}\right), \quad \mathbf{T}^{\alpha}={ }_{\mathrm{o}} \mathbf{T}^{\alpha}(\Delta)+\mathrm{e}^{\alpha}\left(\Delta, \mathbf{a}^{1}, \ldots, \mathbf{a}^{v-1}\right), \\
\pi^{\alpha}={ }_{0} \pi^{\alpha}(\Delta)+\mathrm{e} \pi^{\alpha}\left(\Delta, \mathbf{a}^{1}, \ldots, \mathbf{a}^{v-1}\right),
\end{gathered}
$$

such that the non-equilibrium terms vanish when the relative velocities are zero:

$$
\mathrm{e} \varepsilon^{\alpha}(\Delta, \mathbf{0}, \ldots, \mathbf{0})=0, \quad \mathrm{e} \mathbf{T}^{\alpha}(\Delta, \mathbf{0}, \ldots, \mathbf{0})=\mathbf{0}, \quad \mathrm{e} \pi^{\alpha}(\Delta, \mathbf{0}, \ldots, \mathbf{0})=\mathbf{0}
$$

Further, let

$$
\mathbf{q}^{\alpha}=\hat{\mathbf{q}}^{\alpha}(\Delta, \mathbf{g}), \quad \hat{\mathbf{q}}^{\alpha}(\Delta, \mathbf{0})=\mathbf{0}
$$

In [27], the statement of the Second Law of Thermodynamics extends that presented for thermoelastic materials in [31]. The main difference is that a homothermal process, which is a 
reversible process for a thermoelastic material, may not be a reversible process for a mixture. For example, the diffusion of a fluid through an elastic solid is irreversible. Thus, in [27] it is assumed that a homothermal quasi-static process is a path-independent process. This leads to a prescription for the partial entropy function

$$
\eta^{\alpha}=\hat{\eta}^{\alpha}(\Delta)
$$

where it is assumed that $\eta^{\alpha}$ depends only on the equilibrium state variables $\Delta$ for all processes. Partial Helmholtz free energy functions are defined through

$$
\psi^{\alpha}=\varepsilon^{\alpha}-\theta \eta^{\alpha}
$$

As in (2.20), the free energy functions may be decomposed into equilibrium and non-equilibrium terms:

$$
\psi^{\alpha}={ }_{0} \psi^{\alpha}(\Delta)+\mathrm{e} \psi^{\alpha}\left(\Delta, \mathbf{a}^{1}, \ldots, \mathbf{a}^{v-1}\right), \quad \mathrm{e} \psi^{\alpha}(\Delta, \mathbf{0}, \ldots, \mathbf{0})=0
$$

With (2.20-2.25), and the mixture energy equation (2.17) for homothermal quasi-static processes, the following constitutive results are obtained:

$$
\begin{aligned}
& \eta^{\alpha}=-\frac{\partial_{0} \psi^{\alpha}}{\partial \theta}, \quad{ }_{0} \mathbf{T}^{\alpha}=\sum_{\beta=1}^{v} \rho^{\beta} \frac{\partial_{0} \psi^{\beta}}{\partial \mathbf{F}^{\alpha}} \mathbf{F}^{\alpha^{T}}(\alpha=1, \ldots, v-1), \quad{ }_{\mathrm{o}} \mathbf{T}^{\nu}=-\sum_{\beta=1}^{v} \rho^{\beta} \rho^{\nu} \frac{\partial_{0} \psi^{\beta}}{\partial \rho^{\nu}} \mathbf{1}, \\
& \mathrm{o} \pi^{\alpha}=-\sum_{\beta=1}^{\nu-1}\left(\rho^{\alpha} \frac{\partial \mathrm{o}^{\alpha} \psi^{\alpha}}{\partial \mathbf{F}^{\beta}} \operatorname{grad} \mathbf{F}^{\beta}-\rho^{\beta} \frac{\partial \mathrm{o}^{\beta} \psi^{\beta}}{\partial \mathbf{F}^{\alpha}} \operatorname{grad} \mathbf{F}^{\alpha}\right)-\rho^{\alpha} \frac{\partial \mathrm{o}^{\alpha} \psi^{\alpha}}{\partial \rho^{\nu}} \operatorname{grad} \rho^{\nu}+\rho^{\nu} \frac{\partial \mathrm{o} \psi^{\nu}}{\partial \mathbf{F}^{\alpha}} \operatorname{grad} \mathbf{F}^{\alpha}, \\
& \frac{\partial{ }_{\mathrm{o}} \psi^{\alpha}}{\partial \mathbf{G}^{\beta}}=\frac{\partial_{{ }_{\mathrm{o}}} \psi^{\alpha}}{\partial \operatorname{grad} \rho^{v}}=\mathbf{0} \quad(\alpha=1, \ldots, v ; \beta=1, \ldots, v-1) .
\end{aligned}
$$

These relations also hold for arbitrary processes because none of the variables that appear in (2.26) depend on $\mathbf{a}^{\alpha}$ or $\mathbf{g}$. 
The second part of the Second Law of Thermodynamics is the assumption of an entropy inequality that holds for all processes. Here, the Clausius-Duhem inequality (2.8) as generalized for mixtures is

$$
\sum_{\alpha=1}^{v}\left\{\rho^{\alpha} \frac{\mathrm{d}^{\alpha} \eta^{\alpha}}{\mathrm{dt}}-\frac{\rho^{\alpha} \mathrm{r}^{\alpha}}{\theta}+\operatorname{div}\left(\frac{\mathbf{q}^{\alpha}}{\theta}\right)\right\} \geq 0
$$

this leads to the additional restrictions:

$$
\mathrm{e} \psi^{\alpha}\left(\Delta, \mathbf{a}^{1}, \ldots, \mathbf{a}^{\nu-1}\right)=0, \quad \sum_{\alpha=1}^{v}\left\{\mathrm{e}^{\alpha} \cdot \mathbf{L}^{\alpha}-\mathrm{e} \boldsymbol{\pi}^{\alpha} \cdot \mathbf{a}^{\alpha}\right\}-\frac{\mathbf{q} \cdot \mathbf{g}}{\theta} \geq 0
$$

where $\mathbf{q}=\sum_{\alpha=1}^{v} \mathbf{q}^{\alpha}$. Then, the Helmholtz free energy functions reduce to

$$
\psi^{\alpha}={ }_{\mathrm{o}} \psi^{\alpha}(\Delta)
$$

As noted earlier in this section, there exist mixtures that cannot experience reversible growth processes; thus, an alternative statement of the Second Law of Thermodynamics is necessary. After developing the theory for a growing thermoelastic material in Section 3 without assuming the existence of reversible processes, we extend the approach to mixtures in Section 4.

\subsection{Growing elastic materials}

In this section, the theory of Klisch et al. [12] for growing compressible elastic materials is summarized. The structure of that theory was motivated by how it may be applied in practice. More specifically, tissue explants may be harvested at different stages of the growth process and the tissue's compositional, geometric, and material properties can be experimentally characterized. In order to characterize these properties, the experimental data must be defined relative to a pre-determined reference configuration. Therefore, we introduce a fixed reference 
configuration that can be identified with an experimental configuration of the material, and can be used as a reference configuration for the growth boundary-value problem.

Let $B$ be a growing body with a fixed reference configuration $\kappa_{0}(B)$ and a configuration $\kappa(B)$ at time t. For simplicity, let the body be unloaded and stress-free in $\kappa_{0}(B)^{4}$. The configuration $\kappa(B)$ represents the time-dependent loaded configuration of $B$ during a continuous growth process. A particle of $B$ occupies positions $\mathbf{X} \in \kappa_{0}(B)$ and $\mathbf{x} \in \kappa(B)$. The motion for $B$ is defined as in $(2.1)_{1}$ and is assumed to be invertible. Any material point that is added during the growth process is associated with a unique material point in $\kappa_{0}(B)$ through the inverse mapping of $(2.1)_{1}$.

The deformation gradient of the mapping from $\kappa_{0}(B)$ to $\kappa(B)$ is assumed to obey the decomposition

$$
\mathbf{F}=\mathbf{F}_{l} \mathbf{M}_{e} \mathbf{M}_{g}
$$

The tensor $\mathbf{M}_{e} \mathbf{M}_{g}$ describes the total deformation due to growth relative to $\kappa_{0}(B)$, whereas the deformation gradient $\mathbf{F}_{l}$ represents a superposed elastic deformation caused by applied loads. In the decomposition (2.30), the amount and orientation of mass deposition are described by $\mathbf{M}_{g}$. Furthermore, it is assumed that the mass density, the free energy density, and the stress functions are independent of $\mathbf{M}_{g}$. This latter assumption establishes the existence of the tensors that appear in (2.30). More specifically, $\mathbf{F}_{l}$ can be measured by removing the external loads acting on $\kappa(B)$.

\footnotetext{
${ }^{4}$ In reference [12], it is shown how the theory may be modified to include a residual stress field in the reference configuration $\kappa_{0}(B)$.
} 
Then, $\mathbf{M}_{e} \mathbf{M}_{g}$ can be calculated by measuring the total deformation gradient tensor for $\kappa(B)$ relative to $\kappa(B)$. Finally, $\mathbf{M}_{e}$ and, consequently, $\mathbf{M}_{g}$ can be measured by performing a series of destructive experiments on both $\kappa_{0}(B)$ and $\kappa(B)$ that are designed to relieve the residual stress field in the tissue.

In this theory, the tensors $\mathbf{M}_{e}$ and $\mathbf{M}_{g}$ are introduced relative to a fixed reference configuration and, consequently, lack a clear physical interpretation. However, when interpreted in terms of the continuously changing current configuration of a material during a growth process, these tensors have clear physical meanings. In [12], the governing equations for a small increment of growth were derived in order to provide an intuitive description of the quantities that describe growth. For an increment of growth, the tensor $\mathbf{M}_{g}$ is equivalent to the incremental growth tensor and the tensor $\mathbf{M}_{e}$ is equivalent to the elastic accommodation tensor that ensures compatibility of $\kappa(B)$.

Note that arbitrary, orthogonal tensors $\mathbf{Q}_{1}$ and $\mathbf{Q}_{2}$ may appear in (2.30) such that $\mathbf{F}_{l} \mathbf{M}_{e} \mathbf{M}_{g}=\mathbf{F}_{l} \mathbf{Q}_{1}^{\mathrm{T}} \mathbf{Q}_{1} \mathbf{M}_{e} \mathbf{Q}_{2}^{\mathrm{T}} \mathbf{Q}_{2} \mathbf{M}_{g}$. Epstein and Maugin [33] have discussed invariance requirements when both the present and reference configurations are subject to invariance.

Defining

$$
\mathbf{L}_{l}=\dot{\mathbf{F}}_{l} \mathbf{F}_{l}^{-1}, \quad \mathbf{L}_{e}=\dot{\mathbf{M}}_{e} \mathbf{M}_{e}^{-1}, \quad \mathbf{L}_{g}=\dot{\mathbf{M}}_{g} \mathbf{M}_{g}^{-1},
$$

the velocity gradient tensor associated with $\mathbf{F}$ is

$$
\mathbf{L}=\dot{\mathbf{F}} \mathbf{F}^{-1}=\mathbf{L}_{l}+\mathbf{F}_{l} \mathbf{L}_{e} \mathbf{F}_{l}^{-1}+\mathbf{F}_{l} \mathbf{M}_{e} \mathbf{L}_{g} \mathbf{M}_{e}^{-1} \mathbf{F}_{l}^{-1}
$$


The symmetric parts of $\left(\mathbf{L}_{l}, \mathbf{L}_{e}, \mathbf{L}_{g}\right)$ are denoted as $\left(\mathbf{D}_{l}, \mathbf{D}_{e}, \mathbf{D}_{g}\right)$, respectively. In [12], the following equations were established:

$$
\frac{\dot{\mathbf{J}}}{\mathbf{J}}=\mathbf{D} \cdot \mathbf{1}, \quad \frac{\cdot}{\operatorname{det} \mathbf{M} g}=\mathbf{D} g \cdot \mathbf{1}
$$

The effective elastic deformation gradient tensor $\mathbf{F}_{*}$ and the effective right-Cauchy Green tensor

$\mathbf{C}_{*}$ are defined as

$$
\mathbf{F}_{*}=\mathbf{F}_{l} \mathbf{M}_{e}, \quad \mathbf{C}_{*}=\mathbf{F}_{*}^{\mathrm{T}} \mathbf{F}_{*} .
$$

To state the balance equations, we recall the assumption of [12] that the material deposited during growth has the same mechanical properties as the original material. This assumption has two implications. First, the mass density, linear momentum, angular momentum, internal energy, and kinetic energy of the deposited material are the same as that of the original material at a point. Second, the mechanical response functions of the deposited material are the same as those of the original material. Also, we introduce two scalar parameters. The mass growth function $\mathrm{c}$ is the rate of mass deposition per unit current mass. The growth energy term $\beta$ is the rate of growth energy per unit current mass that is required in addition to that needed to create material with the same internal and kinetic energy as the existing material. Then, the balance equations for mass, linear momentum, angular momentum, and work-energy on $\kappa(B)$ take the form $^{5}$

$$
\dot{\rho}+\rho \operatorname{div} \mathbf{v}=\rho c, \quad \operatorname{div} \mathbf{T}+\rho \mathbf{b}=\rho \dot{\mathbf{v}}, \quad \mathbf{T}=\mathbf{T}^{\mathrm{T}}, \quad \dot{\rho \varepsilon}=\mathbf{T} \cdot \mathbf{D}+\rho \beta .
$$

\footnotetext{
${ }^{5}$ See [12] for these equations stated in integral form.
} 
The mass growth function $\mathrm{c}$ is not a standard variable in the balance of mass equation, and it requires the introduction of an additional equation. Thus, a growth continuity equation is formulated by assuming that the density of the material only depends on the effective elastic deformation tensor. Accordingly, we assume

$$
\rho \mathbf{J}_{*}=\rho_{0},
$$

where $\mathbf{J}_{*}=\operatorname{det} \mathbf{F}_{l} \operatorname{det} \mathbf{M}_{e}$. Combined with $(2.35)_{1}$, this leads to the growth continuity equation

$$
\operatorname{det} \mathbf{M} g=\exp \left[\int_{\tau=\mathrm{t} 0}^{\mathrm{t}} \operatorname{cd} \tau\right]
$$

or, equivalently,

$$
\mathrm{c}=\frac{\frac{\cdot}{\operatorname{det} \mathbf{M} g}}{\operatorname{det} \mathbf{M} g}=\mathbf{D} g \cdot \mathbf{1}
$$

Consider a growing elastic material with constitutive equations

$$
\varepsilon=\tilde{\varepsilon}\left(\mathbf{F}_{*}\right), \quad \mathbf{T}=\tilde{\mathbf{T}}\left(\mathbf{F}_{*}\right) .
$$

Due to invariance requirements, $(2.39)_{1}$ may be written as

$$
\varepsilon=\hat{\varepsilon}\left(\mathbf{C}_{*}\right)
$$

To complete the field equations, a growth law that describes the time-rate of change of $\mathbf{M}_{g}$ must be specified. For the theory to be self-contained, the growth law should depend on mechanical stimuli that are primitive elements of the theory. For example, the growth law may depend on a combination of mechanical stimuli, such as the stress, strain, strain energy, the rate of strain, etc. Since the relationship between growth and mechanical stimuli is poorly understood, here we represent the growth law with the general form 


$$
\dot{\mathbf{M}} g=\hat{G}(M)
$$

where $\hat{\mathcal{G}}(M)$ is an experimentally determined function of mechanical stimuli $M$. Of course, (2.41) must satisfy appropriate invariance requirements. For special forms of the growth law, Epstein and Maugin [33] have derived invariance requirements when both the present and reference configurations are subject to invariance.

For a Green-elastic material, it was shown in [12] that the following constitutive equations may be derived from the work-energy balance $(2.35)_{4}$ :

$$
\mathbf{T}=2 \rho \mathbf{F}_{*} \frac{\partial \hat{\varepsilon}}{\partial \mathbf{C}_{*}} \mathbf{F}_{*}^{\mathrm{T}}, \quad \mathbf{T} \cdot \mathbf{F} l \mathbf{M} e \mathbf{L} g \mathbf{M} e^{-1} \mathbf{F} l^{-1}+\rho \beta=0 .
$$

The result $(2.42)_{2}$ states that the stress power due to growth is balanced by the rate of change of the extra growth energy.

\section{Growing thermoelastic materials}

In this section, we develop a theory for growing thermoelastic materials. The development here combines the theories of thermoelastic materials and growing elastic materials presented in Sections 2.1 and 2.3, respectively. For thermoelastic materials, Casey and Krishnaswamy [31] assumed that a homothermal process is path-independent, or reversible. Here, however, we identify materials that cannot experience reversible growth. Thus, the statement of the Second Law of Thermodynamics presented in [31] is modified.

Here, we construct a family of growing thermoelastic materials that inherit their thermomechanical response functions from a single generating material. This approach is motivated by the idea that, at any time during a continuous growth process, the growth process 
may be stopped. In the theory presented in Section 2, the growth process can be stopped by considering the independent variables $\mathbf{M}_{g}$ to be fixed, so that $\dot{\mathbf{M}}_{g}=\mathbf{0}$. When this occurs, it is evident from (2.38) that $\mathrm{c}=0$ and from $(2.42)_{2}$ that $\beta=0$. Then, experiments may be performed on excised tissue specimens to determine the thermomechanical properties. When the growth process is stopped, the growing material behaves as a thermoelastic material, for which homothermal processes are reversible. This thermoelastic material is called the generating material. Thus, each growing thermoelastic material is associated with a single generating material.

\subsection{Kinematics and balance laws}

Let $B$ be a growing thermoelastic body with a fixed reference configuration $\kappa_{0}(B)$ and a configuration $\kappa(B)$ at time $\mathrm{t}$ (Fig. 1). The temperature history for $B$ is defined as in $(2.1)_{2}$. The kinematics for the growing thermoelastic material are the same as those presented above for the growing elastic material. In particular, relations (2.30-2.34) hold.

Motivated by earlier work, it is assumed that the material deposited during growth has the same thermomechanical properties as the existing material at a point. Then, the balance equations for mass, linear momentum, and angular momentum are the same as $(2.35)_{1,2,3}$. The reduced balance of mass equation (2.36) and the growth continuity equation (2.37) also hold. The balance of energy on $\kappa(B)$ is derived by adding appropriate heating terms to the work-energy balance $(2.35)_{4}$; the resulting equation is:

$$
\rho \dot{\varepsilon}=\rho r-\operatorname{divq}+\mathbf{T} \cdot \mathbf{D}+\rho \beta .
$$


Recalling the expression for the velocity gradient tensor (2.32), the energy equation (3.1) may be expressed as

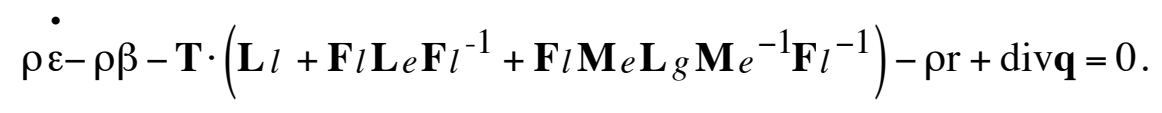

In the purely mechanical theory, the work-energy balance reduced to an equation that allows the determination of $\beta$ from the stress power due to growth; thus, a constitutive equation was not needed for $\beta$. In (3.2), the external heat supply $r$ is an additional unknown; thus, (3.2) may not be used to simultaneously determine $r$ and $\beta$. Consequently, an additional equation is needed. Since the external heat supply may be controlled during experimentation, our approach will be to require a constitutive equation for $\beta$, so that $r$ is determined from (3.2).

\subsection{Growth response functions}

The growth law for the time-rate of change of $\mathbf{M}_{g}$ takes the form (2.41), where the list of stimuli $M$ may include thermal variables, such as temperature or temperature gradient. Also, a growth response function is needed for the growth energy supply $\beta$. Here, we consider growth response functions with the general forms

$$
\dot{\mathbf{M}} g=\hat{G}(M), \quad \beta=\hat{\beta}(M),
$$

where $\hat{\mathcal{G}}(M)$ and $\beta=\hat{\beta}(M)$ are experimentally determined functions of thermomechanical stimuli $M$. It will be seen that the growth response functions (3.3) must obey a constitutive restriction derived from an entropy inequality. As discussed in Section 2.3, (3.3) must satisfy appropriate invariance requirements. 


\subsection{On the existence of reversible processes of growth}

In this section, we establish that there exist thermoelastic materials that cannot experience reversible growth. First, we define a forward process, a reverse process, and a reversible process for growing elastic materials. ${ }^{6}$ Then, a theorem is stated that establishes a necessary condition for reversible growth. The result is used to identify a class of materials that cannot experience reversible growth, and a class of materials that may experience reversible growth.

Attention is restricted to the mechanical aspects of growth under homothermal conditions $(\mathbf{g}=\mathbf{0})$ in the absence of external tractions (so $\mathbf{F}_{l}=\mathbf{0}$ ). Let $P$ be an admissible process from $\mathrm{t}=0$ to $\mathrm{t}=1$; this is called the forward process. Relative to a fixed reference configuration $\kappa_{0}(B)$, the deformation gradient at each time $t$ is (Fig. 2)

$$
\mathbf{F}(\mathrm{t})=\mathbf{M}_{e}(\mathrm{t}) \mathbf{M}_{g}(\mathrm{t}), \quad \mathrm{t} \in[0,1]
$$

Let $\bar{P}$ be the reverse process of $P$; this is specified from $\overline{\mathrm{t}}=0$ to $\overline{\mathrm{t}}=1$, where $\overline{\mathrm{t}}=1$-t. Relative to $\kappa_{0}(B)$, the deformation gradient at each time $\overline{\mathrm{t}}$ is

$$
\overline{\mathbf{F}}(\overline{\mathrm{t}})=\overline{\mathbf{M}}_{e}(\overline{\mathrm{t}}) \overline{\mathbf{M}}_{g}(\overline{\mathrm{t}}), \quad \overline{\mathrm{t}}=1-\mathrm{t}, \quad \overline{\mathrm{t}} \in[0,1] .
$$

Next, we specify the relationship between $P$ and $\bar{P}$ by identifying what conditions must be satisfied in order for $P$ to be reversible. When these conditions are satisfied, $\bar{P}$ is both an admissible and a reversible process.

For a reversible process of an elastic material, the path through elastic strain space (and, consequently, stress space) of the reverse process is the reverse of the path followed for the

\footnotetext{
${ }^{6}$ Here, we follow Erickson [34] who, for a thermoelastic material, used this type of approach to argue that the heat flux vector, $\mathbf{q}$, must vanish in a reversible process.
} 
forward process [34]. However, a growing material experiences both elastic and growth deformations. Thus, the ideas presented by Ericksen [34] concerning reversible processes must be extended in some logical manner to growing elastic materials. The physical idea governing our definition of a reversible process is the following: if $P$ is a reversible process, then, for each point $\mathbf{x}$ and each time t, the configuration and the stress field of an arbitrary neighborhood containing $\mathbf{x}$ in the forward process $P$ is recovered at each corresponding time $\overline{\mathrm{t}}=1-\mathrm{t}$ in the reverse process $\bar{P}$. Since we will assume that the Cauchy stress depends only on $\mathbf{M}_{e}$ in the absence of external loading, the following definition is used:

Definition 1: The process $P$ is reversible if

(i) $\mathbf{F}(\mathrm{t})=\overline{\mathbf{F}}(\overline{\mathrm{t}})$ for all $\mathrm{t} \in[0,1]$; and

(ii) $\mathbf{M}_{e}(\mathrm{t})=\overline{\mathbf{M}}_{e}(\overline{\mathrm{t}})$ for all $\mathrm{t} \in[0,1]$.

With the additional assumption that $\mathbf{M}_{g}(\mathrm{t})$ and its time-derivatives are continuous in the interval $[0,1]$, we have:

Theorem: A necessary condition for $P$ to be reversible is that

$$
\dot{\overline{\mathbf{M}}}_{g}(\overline{\mathrm{t}})=-\dot{\mathbf{M}}_{g}(\mathrm{t}) \text { for all } \mathrm{t} \in[0,1] \text {. }
$$

The proof is in the appendix.

With this theorem, it is now possible to identify a class of materials which cannot experience reversible growth. Consider a material with a growth response function of the form:

$$
\dot{\mathbf{M}}_{g}=\hat{\mathcal{G}}\left(\mathbf{T}, \mathbf{M}_{e}, \mathbf{M}_{g}\right) \text {. }
$$

Recalling $(2.39)_{2}$, assume that the Cauchy stress obeys a constitutive equation of the type 


$$
\mathbf{T}(\mathrm{t})=\tilde{\mathbf{T}}\left(\mathbf{M}_{\mathrm{e}}(\mathrm{t})\right) .
$$

For a reversible process, Definition 1 (ii), (A.2), and (3.7) yield

$$
\mathbf{M}_{e}(\mathrm{t})=\overline{\mathbf{M}}_{e}(\overline{\mathrm{t}}), \quad \mathbf{M}_{g}(\mathrm{t})=\overline{\mathbf{M}}_{g}(\overline{\mathrm{t}}), \quad \mathbf{T}(\mathrm{t})=\overline{\mathbf{T}}(\overline{\mathrm{t}}) .
$$

Combined with (3.6), this gives

$$
\dot{\overline{\mathbf{M}}}_{g}(\overline{\mathrm{t}})=\hat{\mathcal{G}}\left(\overline{\mathbf{T}}(\overline{\mathrm{t}}), \overline{\mathbf{M}}_{e}(\overline{\mathrm{t}}), \overline{\mathbf{M}}_{g}(\overline{\mathrm{t}})\right)=\hat{\mathcal{G}}\left(\mathbf{T}(\mathrm{t}), \mathbf{M}_{e}(\mathrm{t}), \mathbf{M} g(\mathrm{t})\right)=\dot{\mathbf{M}}_{g}(\mathrm{t}),
$$

which does not satisfy the necessary condition stated in the theorem. Therefore, the class of materials which obey (3.6-3.7) cannot experience reversible growth.

It is also possible to identify a class of materials for which reversible growth is possible. Consider a growth response function that depends on the elastic strain rate:

$$
\dot{\mathbf{M}}_{g}=\hat{\mathcal{G}}\left(\mathbf{T}, \mathbf{M}_{e}, \mathbf{M}_{g}, \dot{\mathbf{M}}_{e}\right) \text {. }
$$

Then, it is easy to show that a reversible process exists if (3.10) satisfies the restriction:

$$
\hat{\mathcal{G}}\left(\mathbf{T}, \mathbf{M}_{e}, \mathbf{M}_{g},-\dot{\mathbf{M}}_{e}\right)=-\hat{\mathcal{G}}\left(\mathbf{T}, \mathbf{M}_{e}, \mathbf{M}_{g}, \dot{\mathbf{M}}_{e}\right)
$$

Thus, only a subset of materials with the rate-dependent growth functions (3.10) may experience reversible growth.

It is now clear that there exist growing thermoelastic materials that cannot experience reversible growth. Therefore, the application of the Second Law of Thermodynamics for thermoelastic materials presented in [31] must be modified.

\subsection{Construction of a family of growing thermoelastic materials}

In this section, a growing thermoelastic material is constructed from a generating material. Recall that the material deposited during growth is assumed to have the same thermomechanical 
properties as the original material at a point, and that the tensor $\mathbf{M}_{g}$ describes the amount and orientation of mass deposition. Consequently, we assume that a growing thermoelastic material $m_{\mathrm{g}}$ inherits its response functions for free energy, stress, entropy, and heat flux from those of a generating material $m$ (i.e., a unique thermoelastic material), with one exception: the response functions for $m_{\mathrm{g}}$ are evaluated at the effective elastic deformation gradient, i.e., at $\mathbf{F}=\mathbf{F}_{*}$. In particular, the response functions for each growing thermoelastic material $m_{\mathrm{g}}$ are obtained from a generating material $m$ as follows:

$$
\begin{array}{ll}
\hat{\psi}_{m_{\mathrm{g}}}(\mathbf{C} *, \theta)=\hat{\psi}_{m}(\mathbf{C}, \theta), \quad \tilde{\mathbf{T}}_{m_{\mathrm{g}}}\left(\mathbf{F}_{*}, \theta\right)=\tilde{\mathbf{T}}_{m}(\mathbf{F}, \theta), \quad \tilde{\mathbf{q}}_{m_{\mathrm{g}}}(\mathbf{F} *, \theta, \mathbf{g})=\tilde{\mathbf{q}}_{m}(\mathbf{F}, \theta, \mathbf{g}), \\
\hat{\eta}_{m_{\mathrm{g}}}\left(\mathbf{C}_{*}, \theta\right)=\hat{\eta}_{m}(\mathbf{C}, \theta) .
\end{array}
$$

Then, recalling (2.5-2.7), we obtain

$$
\tilde{\mathbf{T}}_{m_{\mathrm{g}}}(\mathbf{F} *, \theta)=2 \rho \mathbf{F} * \frac{\partial \hat{\psi}_{m_{\mathrm{g}}}}{\partial \mathbf{C}_{*}} \mathbf{F}_{*}^{\mathrm{T}}, \quad \hat{\boldsymbol{\eta}}_{m_{\mathrm{g}}}\left(\mathbf{C}_{*}, \theta\right)=-\frac{\partial \hat{\psi}_{m_{\mathrm{g}}}}{\partial \theta} .
$$

Thus, for a growing thermoelastic material the entropy function is prescribed by considering homothermal processes of the generating material. Also, each growing thermoelastic material is associated with a single generating material. Note that (3.12) $)_{1,4}$ combined with (2.6) furnishes a response function for the internal energy of a growing thermoelastic material.

An equivalence relation on the set of all growing thermoelastic materials is defined as follows: $m_{\mathrm{g} 1}$ is equivalent to $m_{\mathrm{g} 2}\left(m_{\mathrm{g} 1} \sim m_{\mathrm{g} 2}\right)$ if and only if their response functions (3.12) are equal for all values of $\left(\mathbf{F}_{*}, \theta, \mathbf{g}\right)$. Thus, each thermoelastic material $m$ generates an equivalence class, or family, of growing thermoelastic materials $m_{\mathrm{g}}$ which share the same response functions 
listed in (3.12), while having distinct growth response functions for $\dot{\mathbf{M}}_{g}$ and $\beta$. In the next

section, a constitutive restriction relating $\dot{\mathbf{M}}_{g}$ and $\beta$ is derived using an entropy inequality.

\subsection{Restrictions imposed by the Second Law of Thermodynamics}

Here, only growing thermoelastic materials are considered and, for convenience, the subscript $m_{\mathrm{g}}$ is dropped from (3.12-3.13). With (2.6) and (3.12-3.13), the energy equation for $m_{\mathrm{g}}$ (3.2) reduces to

$$
\dot{\rho \eta} \theta=\rho \mathrm{r}-\operatorname{divq}+\left\{\mathbf{M}_{e}^{\mathrm{T}} \mathbf{F}_{l}^{\mathrm{T}} \mathbf{T F}_{l}^{-\mathrm{T}} \mathbf{M}_{e}^{-\mathrm{T}}\right\} \cdot \mathbf{L}_{g}+\rho \beta
$$

Now, an entropy inequality for a growing material is developed by generalizing the ClausiusDuhem inequality. Consider an arbitrary region $\mathcal{P}$ fixed in $\mathcal{E}^{3}$ and bounded by a closed surface $\partial \mathcal{P}$ with unit outward normal $\mathbf{n}$. The integral form of this entropy inequality is

$$
\frac{\partial}{\partial \mathrm{t}} \int_{\mathcal{P}} \rho \eta d V+\int_{\partial \mathcal{P}} \rho \eta \mathbf{v} \cdot \mathbf{n} d A \geq \int_{\mathcal{P}} \rho \frac{\mathrm{r}}{\theta} d V-\int_{\partial \mathcal{P}} \frac{1}{\theta} \mathbf{q} \cdot \mathbf{n} d A+\int_{\mathcal{P}} \rho \mathrm{c} \eta d V
$$

Recalling the assumption that the material deposited has the same thermomechanical properties as the existing material, the entropy per unit mass of the deposited material is taken to equal that of the existing material. This is represented by the last integral in (3.15). With the balance of mass $(2.35)_{1}$, the local form of the Clausius-Duhem inequality is obtained:

$$
\rho \dot{\eta}-\rho \frac{\mathrm{r}}{\theta}+\operatorname{div} \frac{\mathbf{q}}{\theta} \geq 0
$$

With (3.14), (3.16) becomes

$$
\left\{\mathbf{M}_{e}{ }^{\mathrm{T}} \mathbf{F}_{l}{ }^{\mathrm{T}} \mathbf{T} \mathbf{F}_{l}{ }^{-\mathrm{T}} \mathbf{M}_{e}{ }^{-\mathrm{T}}\right\} \cdot \mathbf{L}_{g}+\rho \beta-\frac{\mathbf{q} \cdot \mathbf{g}}{\theta} \geq 0
$$


For homothermal processes $(\mathbf{g}=\mathbf{0}),(3.17)$ reduces to

$$
\mathbf{T} \cdot \mathbf{F}_{l} \mathbf{M}_{e} \dot{\mathbf{M}}_{g} \mathbf{M}_{g}^{-1} \mathbf{M}_{e}^{-1} \mathbf{F}_{l}^{-1}+\rho \beta \geq 0
$$

This inequality is the constitutive restriction that the growth response functions $\dot{\mathbf{M}}_{g}$ and $\beta$ must satisfy for homothermal processes.

\section{Mixtures of growing materials}

In this section, a theory of growth for mixtures of an arbitrary number of thermoelastic materials and a single fluid is constructed. Most of the development that follows is the logical extension of the theories presented above for a mixture (Section 2.2) and a growing thermoelastic material (Section 3). The major difference lies in identifying the balance equations for the growing mixture. Many authors have studied mixtures in which the constituent mass may change through internal reactions; e.g., see Atkin and Craine [35]. In those theories, the balance of mass for each constituent includes a mass supply term that is conceptually different from the mass growth function c presented in Section 2.3. This is due to the assumption that the total mixture mass is conserved in those theories with internal mass exchange, whereas the total mixture mass will typically change for a growing mixture. Consequently, the balance equations derived for a growing mixture are different.

\subsection{Kinematics}

Consider a mixture $B$ of $v$ constituents: $v-1$ growing elastic materials $C^{\alpha}(\alpha \in[1, v-1])$ and an inviscid fluid $C^{v}$. Let $\kappa_{0}(B)$ be a fixed reference configuration and $\kappa(B)$ a time-dependent 
loaded configuration at time t. The motion and temperature histories for a growing mixture are defined as in (2.10).

The kinematics for each growing elastic constituent $C^{\alpha}$ are the same as that adopted for the growing elastic material. In particular, the deformation gradient tensor for each growing elastic material obeys the decomposition

$$
\mathbf{F}^{\alpha}=\mathbf{F}_{l}^{\alpha} \mathbf{M}_{e}^{\alpha} \mathbf{M}_{g}^{\alpha}, \quad \alpha \in[1, v-1]
$$

Also, the relations (2.31-2.34) hold for the kinematics of each growing elastic material.

\subsection{Balance Laws}

The balance laws for a mixture presented in Section 2.2 are modified to include variables associated with mass deposition and growth energy supply. Here, the mass growth function $\mathrm{c}^{\alpha}$ and the growth energy density supply $\beta^{\alpha}$ are defined for each growing elastic material. Then, the balance equations for mass, linear momentum, angular momentum, and energy on $\kappa(B)$ are

$$
\begin{gathered}
\frac{d^{\alpha} \rho^{\alpha}}{d t}+\rho^{\alpha} \operatorname{div} \mathbf{v}^{\alpha}=\rho^{\alpha} c^{\alpha},(\alpha \in[1, v-1]) ; \quad \frac{d^{v} \rho^{v}}{d t}+\rho^{v} \operatorname{div} \mathbf{v}^{v}=0, \\
\rho^{\alpha} \frac{d^{\alpha} \mathbf{v}^{\alpha}}{d t}=\operatorname{div}^{\alpha}+\pi^{\alpha}+\rho^{\alpha} \mathbf{b}^{\alpha}, \\
\mathbf{T}^{\alpha}-\mathbf{T}^{\alpha^{T}}=\Lambda^{\alpha}, \\
\rho^{\alpha} \frac{d^{\alpha} \varepsilon}{d t}=\rho^{\alpha} r^{\alpha}-\operatorname{divq}^{\alpha}+\gamma^{\alpha}+\mathbf{T}^{\alpha} \cdot \mathbf{D}^{\alpha}+\rho^{\alpha} \beta^{\alpha}, \quad(\alpha \in[1, v-1]) ; \\
\rho^{v} \frac{d^{v} \varepsilon^{v}}{d t}=\rho^{v} r^{v}-\operatorname{div} \mathbf{q}^{v}+\gamma^{v}+\mathbf{T}^{v} \cdot \mathbf{D}^{v} .
\end{gathered}
$$


Assuming that the change in density of each growing elastic material $C^{\alpha}$ is due only to the elastic part of the total deformation of $C^{\alpha}$, the continuity and growth continuity equations for each $C^{\alpha}$ take the form (2.36-2.37).

When stating the integral forms for the balance equations of the mixture, terms are included that account for the net change of mass, linear momentum, angular momentum, and energy of the mixture due to growth. Then, the balance of mass, linear momentum, and angular momentum equations for the mixture require that

$$
\sum_{\alpha=1}^{v-1} \rho^{\alpha} c^{\alpha}=\rho c, \quad \sum_{\alpha=1}^{v} \pi^{\alpha}=\mathbf{0}, \quad \sum_{\alpha=1}^{v} \Lambda^{\alpha}=\mathbf{0}
$$

where $\mathrm{c}$ is the mass growth function for the mixture. The balance of energy equation for the mixture becomes

$$
\sum_{\alpha=1}^{v}\left[\rho^{\alpha} \frac{\mathrm{d}^{\alpha} \varepsilon^{\alpha}}{\mathrm{dt}}-\rho^{\alpha} \mathrm{r}^{\alpha}+\operatorname{div} \mathbf{q}^{\alpha}+\pi^{\alpha} \cdot \mathbf{v}^{\alpha}-\mathbf{T}^{\alpha} \cdot \mathbf{L}^{\alpha}\right]-\sum_{\alpha=1}^{v-1} \rho^{\alpha} \beta^{\alpha}=0 .
$$

In the mixture theories that allow internal mass exchange, the mixture balance equations are different. For example, the mixture balance of linear momentum corresponding to (4.6) $)_{2}$ appears as $\sum_{\alpha=1}^{v}\left(\pi^{\alpha}+c^{\alpha} \mathbf{v}^{\alpha}\right)=\mathbf{0} \quad[35] .{ }^{7}$ The integral forms of those balance equations are derived by assuming that the mass that is transferred between constituents does not contribute to the total linear momentum, angular momentum, and energy of the mixture. This assumption is based on the requirement that the total mixture mass is conserved. Here, the deposited mass does

\footnotetext{
${ }^{7}$ The term $\mathbf{J}^{\alpha}$ that appears in [35] is equal to $\mathbf{v}^{\alpha}$ since the velocity of the mass created at any point is assumed to equal the velocity of the mass that exists at that point.
} 
contribute to the integral forms of the balance equations, since the total mass of the mixture will typically change during the growth process.

\subsection{Growth response functions}

The growth response functions for the material time-derivatives of $\mathbf{M}_{g}^{\alpha}$ and $\beta^{\alpha}$ for each growing elastic constituent $C^{\alpha}$ are generalized from (3.3). Thus, consider growth response functions with the general forms

$$
\frac{\mathrm{d}^{\alpha} \mathbf{M}_{g}^{\alpha}}{\mathrm{dt}}=\hat{\mathcal{G}}^{\alpha}\left(M^{\alpha}\right), \quad \beta^{\alpha}=\hat{\beta}^{\alpha}\left(M^{\alpha}\right),
$$

where $C^{\alpha}$ represents the thermomechanical stimuli that drive the growth process for each $C^{\alpha}$. Since growth may be modulated by fluid diffusion in cartilage [36], the relative fluid velocity may be included.

\subsection{Construction of a family of growing thermoelastic materials}

A growing mixture $m_{\mathrm{g}}$ is assumed to inherit its response functions for partial free energy, partial stress, diffusive force, partial entropy, and partial heat flux vector from those of a generating mixture $m$, with an exception: the response functions for $m_{\mathrm{g}}$ are evaluated at the

effective elastic deformation gradient tensors, i.e., at $\mathbf{F}^{\alpha}=\mathbf{F}_{*}^{\alpha}$ and $\mathbf{G}^{\alpha}=\mathbf{G}_{*}^{\alpha}$. Thus, for $m_{\mathrm{g}}$ let

$$
\Delta *=\left\{\mathbf{F}_{*}^{1}, \ldots, \mathbf{F}_{*}^{\nu-1}, \rho^{\nu}, \mathbf{G}_{*}^{1}, \ldots, \mathbf{G}_{*}^{\nu-1}, \operatorname{grad} \rho^{\nu}, \theta\right\}
$$

Then, the response functions for each growing mixture $m_{\mathrm{g}}$ are obtained from a generating mixture $m$ such that 


$$
\begin{aligned}
& \psi_{m_{\mathrm{g}}}^{\alpha}\left(\Delta_{*}\right)=\psi_{m}^{\alpha}(\Delta),{ }_{\mathrm{o}} \mathbf{T}_{m_{\mathrm{g}}}^{\alpha}\left(\Delta_{*}\right)={ }_{\mathrm{o}} \mathbf{T}_{m}^{\alpha}(\Delta), \quad \mathrm{e}_{m_{\mathrm{g}}}^{\alpha}\left(\Delta_{*}, \mathbf{a}^{1}, \ldots, \mathbf{a}^{\nu-1}\right)={ }_{\mathrm{e}} \mathbf{T}_{m}^{\alpha}\left(\Delta, \mathbf{a}^{1}, \ldots, \mathbf{a}^{\nu-1}\right),
\end{aligned}
$$

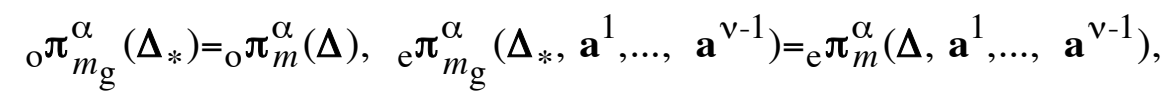

$$
\begin{aligned}
& \eta_{m_{\mathrm{g}}}^{\alpha}\left(\Delta_{*}\right)=\eta_{m}^{\alpha}(\Delta), \quad \mathbf{q}_{m_{\mathrm{g}}}^{\alpha}\left(\Delta_{*}, \mathbf{g}\right)=\mathbf{q}_{m}^{\alpha}(\Delta, \mathbf{g})
\end{aligned}
$$

where $\Delta$ is defined in (2.19). When applied to (4.10), (2.26) and (2.28) yield

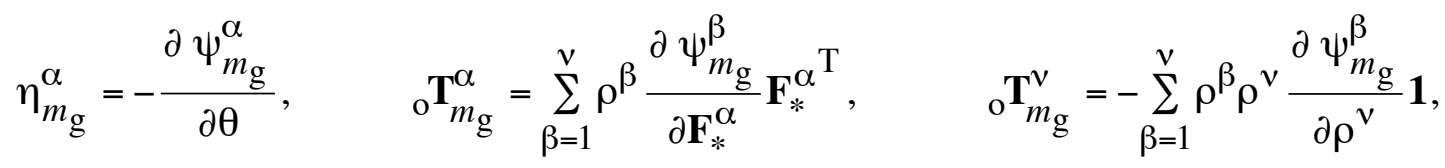

$$
\begin{aligned}
& { }_{\mathrm{o}} \boldsymbol{\pi}_{m_{\mathrm{g}}}^{\alpha}=-\sum_{\beta=1}^{v-1}\left(\rho^{\alpha} \frac{\partial \psi_{m_{\mathrm{g}}}^{\alpha}}{\partial \mathbf{F}_{*}^{\beta}} \operatorname{grad} \mathbf{F}_{*}^{\beta}-\rho^{\beta} \frac{\partial \psi_{m_{\mathrm{g}}}^{\beta}}{\partial \mathbf{F}_{*}^{\alpha}} \operatorname{grad}_{*}^{\alpha}\right) \\
& -\rho^{\alpha} \frac{\partial \psi_{m_{\mathrm{g}}}^{\alpha}}{\partial \rho^{v}} \operatorname{grad} \rho^{v}+\rho^{\nu} \frac{\partial \psi_{m_{\mathrm{g}}}^{v}}{\partial \mathbf{F}_{*}^{\alpha}} \operatorname{grad} \mathbf{F}_{*}^{\alpha}
\end{aligned}
$$

An equivalence relation on the set of all growing mixtures may be defined as in Section 3 so that two equivalent growing mixtures differ only through their growth response functions (4.8).

\subsection{Restrictions imposed by the Second Law of Thermodynamics}

Here, we consider only growing mixtures and, for convenience, drop the subscript $m_{\mathrm{g}}$ from

the above equations. With $(2.20),(2.24-2.26),(2.32)$, and (4.11) the energy equation for $m_{\mathrm{g}}(4.7)$

reduces to

$$
\begin{array}{r}
\sum_{\alpha=1}^{v-1}\left\{\rho^{\alpha} \theta \frac{d^{\alpha} \eta^{\alpha}}{d t}-\rho^{\alpha} r^{\alpha}+\operatorname{div} \mathbf{q}^{\alpha}+{ }_{e} \pi^{\alpha} \cdot \mathbf{a}^{\alpha}-{ }_{0} \mathbf{T}^{\alpha} \cdot \mathbf{F}_{*}^{\alpha} \mathbf{L}_{g}^{\alpha} \mathbf{F}_{*}^{\alpha^{-1}}\right. \\
\left.-{ }_{\mathrm{e}} \mathbf{T}^{\alpha} \cdot \mathbf{L}^{\alpha}-\rho^{\alpha} \beta^{\alpha}\right\}+\rho^{v} \theta \frac{d^{v} \eta^{v}}{d t}-\rho^{v} r^{v}+\operatorname{div} \mathbf{q}^{v}-{ }_{e} \mathbf{T}^{v} \cdot \mathbf{L}^{v}=0 .
\end{array}
$$

The Clausius-Duhem inequality is generalized for a growing mixture following (3.15-3.16); the result is 


$$
\sum_{\alpha=1}^{v}\left\{\rho^{\alpha} \frac{\mathrm{d}^{\alpha} \eta^{\alpha}}{\mathrm{dt}}-\frac{\rho^{\alpha} \mathrm{r}^{\alpha}}{\theta}+\operatorname{div}\left(\frac{\mathbf{q}^{\alpha}}{\theta}\right)\right\} \geq 0
$$

Using (4.12), the entropy inequality becomes

$$
\sum_{\alpha=1}^{v-1}\left\{{ }_{0} \mathbf{T}^{\alpha} \cdot \mathbf{F}_{*}^{\alpha} \mathbf{L}_{g}^{\alpha} \mathbf{F}_{*}^{\alpha^{-1}}+{ }_{e} \mathbf{T}^{\alpha} \cdot \mathbf{L}^{\alpha}-{ }_{e} \pi^{\alpha} \cdot \mathbf{a}^{\alpha}+\rho^{\alpha} \beta^{\alpha}\right\}+{ }_{e} \mathbf{T}^{v} \cdot \mathbf{L}^{\nu}-\frac{\mathbf{q} \cdot \mathbf{g}}{\theta} \geq 0
$$

\section{A cartilage growth model}

A cartilage growth model may be derived by specializing the theory presented in Section 4. In particular, the model consists of a mixture of two growing elastic materials $(\alpha=1,2)$, that represent the proteoglycan and collagen constituents, and a single fluid, which represents the water and dissolved solutes. Also, the model includes two special types of mechanical constraints that are relevant to cartilage. The first constraint states that the deformation gradient tensors $\mathbf{F}^{\alpha}$ of the growing proteoglycan and collagen constituents are equal. In most cases, this may be a reasonable assumption as most of the proteoglycans $(\sim 60-80 \%)$ and the collagens ( $95 \%)$ are effectively immobilized in the tissue matrix [37-39]. Thus, when neglecting the mechanical effect of the mobile molecules, the total deformation gradient tensors of the proteoglycan and collagen constituents are equal at every point. Of course, the theory presented in Section 4 is general enough to allow for mobile constituents. For example, the solid matrix can be modeled as being composed of four constituents: two that represent the proteoglycan and collagen molecules that are bound to the extracellular matrix, and two that represent the mobile proteoglycan and collagen molecules. The second constraint is the internal constraint of intrinsic 
incompressibility [40] that is commonly employed in the study of cartilage mechanics [41]. This constraint has demonstrated experimentally for physiologic levels of hydrostatic pressure [42].

A procedure first introduced by Adkins [43] and further developed by Truesdell and Noll [44] for introducing internal constraints in finite elasticity is used. For a growing mixture subject to a mechanical constraint, the partial stresses and diffusive forces are assumed to obey the additive decompositions

$$
\mathbf{T}^{\alpha}=\overline{\mathbf{T}}^{\alpha}+\hat{\mathbf{T}}^{\alpha}, \quad \pi^{\alpha}=\bar{\pi}^{\alpha}+\hat{\pi}^{\alpha},
$$

where $\left(\overline{\mathbf{T}}^{\alpha}, \overline{\boldsymbol{\pi}}^{\alpha}\right)$ are indeterminate partial stresses and diffusive forces called the constraint responses, and $\left(\hat{\mathbf{T}}^{\alpha}, \hat{\boldsymbol{\pi}}^{\alpha}\right)$ are determinate partial stresses and diffusive forces. ${ }^{8}$ Furthermore, the work done by the constraint response functions is assumed to equal zero:

$$
\sum_{\alpha=1}^{v}\left(\overline{\mathbf{T}}^{\alpha} \cdot \mathbf{L}^{\alpha}-\bar{\pi}^{\alpha} \cdot \mathbf{a}^{\alpha}\right)=0 .
$$

\subsection{Equal total deformation gradient tensors}

The requirement that the overall deformation gradient tensors of the two growing elastic materials be identical results in the nine constraint equations

$$
\phi_{\mathrm{ij}}=\mathbf{e}_{\mathrm{i}} \cdot \mathbf{F}^{1} \mathbf{e}_{\mathrm{j}}-\mathbf{e}_{\mathrm{i}} \cdot \mathbf{F}^{2} \mathbf{e}_{\mathrm{j}}=0, \quad(\mathrm{i}, \mathrm{j}=1,2,3)
$$

where $\left(\mathbf{e}_{1}, \mathbf{e}_{2}, \mathbf{e}_{3}\right)$ is a fixed orthonormal basis. Taking the material time derivative with respect to $C^{1}$ and introducing the resulting nine equations each multiplied by a Lagrange multiplier into the condition (5.2) results in

\footnotetext{
${ }^{8}$ Adkins [43] also assumed that the indeterminate response for the stress was symmetric, whereas Truesdell and Noll [44] provided a geometric argument to obtain the constraint response and proved that the indeterminate stress must be symmetric for a finitely elastic material.
} 


$$
\left(\overline{\mathbf{T}}^{1}+\lambda \mathbf{F}^{1^{\mathrm{T}}}\right) \cdot \mathbf{L}^{1}+\left(\overline{\mathbf{T}}^{2}-\lambda \mathbf{F}^{2^{\mathrm{T}}}\right) \cdot \mathbf{L}^{2}-\overline{\boldsymbol{\pi}}^{1} \cdot \mathbf{a}^{1}-\overline{\boldsymbol{\pi}}^{2} \cdot \mathbf{a}^{2}=0
$$

for all ( $\left.\mathbf{L}^{1}, \mathbf{L}^{2}, \mathbf{a}^{1}, \mathbf{a}^{2}\right)$ satisfying the constraint (5.3), where $\lambda$ is an arbitrary second-order tensor. However, due to the constraint (5.3), $\mathbf{L}^{1}=\mathbf{L}^{2}$ and $\mathbf{a}^{1}=\mathbf{a}^{2}$; consequently, (5.4) produces the result

$$
\overline{\mathbf{T}}^{1}=-\overline{\mathbf{T}}^{2}=\bar{\lambda}, \quad \overline{\boldsymbol{\pi}}^{1}=-\overline{\boldsymbol{\pi}}^{2}=\overline{\mathbf{p}},
$$

where $\bar{\lambda}$ and $\overline{\mathbf{p}}$ are arbitrary.

\subsection{Intrinsic incompressibility}

For intrinsic incompressibility, each constituent $C^{\alpha}$ is assumed to be separable from the others with constant (true) density $\rho^{\alpha \mathrm{T}}$ defined as the mass of $C^{\alpha}$ per unit volume of $C^{\alpha}$. It is assumed that the volume of the mixture is equal to the sum of the volumes of each $C^{\alpha}$. With these assumptions, Mills [40] derived the constraint equation:

$$
\phi=\sum_{\alpha=1}^{3}\left\{\frac{\rho^{\alpha}}{\rho^{\alpha \mathrm{T}}}\right\}-1=0 .
$$

Taking the material time derivative with respect to the fluid constituent $(\alpha=3)$, recalling (2.13), (2.31), (2.38), and (4.2) 1 , and introducing the resulting equation multiplied by a Lagrange multiplier p into (5.2) yields

$$
\begin{aligned}
\sum_{\alpha=1}^{2}\left\{\left(\overline{\mathbf{T}}^{\alpha}-\mathrm{p} \frac{\rho^{\alpha}}{\rho^{\alpha \mathrm{T}}} \mathbf{1}\right) \cdot \mathbf{L}_{l}^{\alpha}+\left(\mathbf{F}_{l}^{\alpha^{\mathrm{T}}} \overline{\mathbf{T}}^{\alpha} \mathbf{F}_{l}^{\alpha^{-\mathrm{T}}}-\mathrm{p} \frac{\rho^{\alpha}}{\rho^{\alpha \mathrm{T}}} \mathbf{1}\right) \cdot \mathbf{L}_{e}^{\alpha}\right. \\
\left.+\overline{\mathbf{T}}^{\alpha} \cdot \mathbf{F}_{l}^{\alpha} \mathbf{M}_{e}^{\alpha} \mathbf{L}_{g}^{\alpha} \mathbf{M}_{e}^{\alpha^{-1}} \mathbf{F}_{l}^{\alpha^{-1}}-\left(\overline{\boldsymbol{\pi}}^{\alpha}+\mathrm{p} \frac{\operatorname{grad} \rho^{\alpha}}{\rho^{\alpha \mathrm{T}}}\right) \cdot \mathbf{a}^{\alpha}\right\} \\
+\left(\overline{\mathbf{T}}^{3}-\mathrm{p} \frac{\rho^{3}}{\rho^{3 \mathrm{~T}}} \mathbf{1}\right) \cdot \mathbf{L}^{3}=0 .
\end{aligned}
$$


Since $\mathbf{L}_{l}^{\alpha}$ may be chosen independently from $\left(\mathbf{L}_{e}^{\beta}, \mathbf{L}_{g}^{\beta}, \mathbf{L}^{3}, \mathbf{a}^{\beta}\right)$, it follows that the indeterminate partial stresses must satisfy

$$
\overline{\mathbf{T}}^{\alpha}=\mathrm{p} \frac{\rho^{\alpha}}{\rho^{\alpha \mathrm{T}}} \mathbf{1}
$$

while the indeterminate diffusive forces must satisfy (recalling (2.38))

$$
\sum_{\alpha=1}^{2}\left\{p \frac{\rho^{\alpha}}{\rho^{\alpha \mathrm{T}}} c^{\alpha}-\left(\bar{\pi}^{\alpha}+p \frac{\operatorname{grad} \rho^{\alpha}}{\rho^{\alpha \mathrm{T}}}\right) \cdot \mathbf{a}^{\alpha}\right\}=0 .
$$

Recalling (2.38), note that $\mathrm{c}^{\alpha}$ is obtained from the constitutive equation for the material time-

derivative $\mathrm{d}^{\alpha}(\cdot) / \mathrm{dt}$ of $\mathbf{M}_{g}^{\alpha}(4.8)_{1}$ which, in general, may depend on the relative velocities $\mathbf{a}^{\beta}$.

Thus, for the general case no further reduction is possible for (5.9). However, it seems plausible to suggest that $\mathrm{c}^{\alpha}$ only depends on the magnitude of the relative velocities. Thus, consider the following special assumption concerning the derived response function for each $c^{\alpha}(\alpha=1, v-1)$ :

$$
c^{\alpha}\left(\mathbf{a}^{\beta}\right)=c^{\alpha}\left(-\mathbf{a}^{\beta}\right), \quad(\beta=1, v-1)
$$

For this special assumption, (5.9) reveals that the indeterminate diffusive forces must satisfy

$$
\bar{\pi}^{\alpha}=-p \frac{\operatorname{grad} \rho^{\alpha}}{\rho^{\alpha \mathrm{T}}}
$$

\section{Discussion}

In this paper we developed a theory of volumetric growth for a mixture of an arbitrary number of elastic materials and an inviscid fluid. As noted in the Introduction, this work is an important step towards developing models that describe the mechanics of the growth process in 
cartilage. A key feature of the theory is that the individual constituents can grow independently which leads to remodeling of the tissue while the response functions of the constituents remain unchanged. In initial applications of this theory to study the growth of cartilage, we plan to model the cartilage as a mixture of two growing elastic materials and an inviscid fluid, subject to the two internal constraints outlined in Section 5.

A mechanical model of cartilage growth can be used in a number of ways. It may be used to identify the manner in which tissue-engineered cartilage constructs may be stimulated in vitro for the fabrication of better repair implants. In particular, the design of tissue-engineered constructs may benefit from the identification of requirements for the molecular composition and the mechanical properties that are needed for the cartilage implant to successfully integrate with the surrounding tissue. A cartilage growth model could also be used to design computer models of the in vivo growth, degeneration, and repair processes, which can be used as predictive tools for the design of therapies for the successful repair of growth and degenerative abnormalities. This type of approach can be useful much in the same manner that finite element analysis has been for a number of design problems in bioengineering, such as the successful design of hip and knee prostheses that meet requirements due to function, wear, and life expectancy.

\section{Acknowledgments}

The authors are grateful for support from the U.C. President's Office Postdoctoral Fellowship Program (SMK), grant 1F32AR08577-01 from NIH (SMK), and grant CMS 9634903 from NSF (AH). 


\section{Appendix}

In Section 3.3, the following theorem for growing elastic materials was stated:

Theorem: A necessary condition for $P$ to be reversible is that

$$
\dot{\overline{\mathbf{M}}}_{g}(\overline{\mathrm{t}})=-\dot{\mathbf{M}}_{g}(\mathrm{t}) \text { for all } \mathrm{t} \in[0,1]
$$

The proof is as follows.

Assume that $P$ is reversible. By Definition 1 (i) and (3.4-3.5),

$$
\mathbf{M}_{e}(\mathrm{t}) \mathbf{M}_{g}(\mathrm{t})=\overline{\mathbf{M}}_{e}(\overline{\mathrm{t}}) \overline{\mathbf{M}}_{g}(\overline{\mathrm{t}}) .
$$

Since $\mathbf{M}_{e}(\mathrm{t})=\overline{\mathbf{M}}_{e}(\overline{\mathrm{t}})$ by Definition 1 (ii) and $\mathbf{M}_{e}(\mathrm{t})$ is invertible, this yields

$$
\mathbf{M}_{g}(\mathrm{t})=\overline{\mathbf{M}}_{g}(\overline{\mathrm{t}}) \text { for all } \mathrm{t} \in[0,1]
$$

Pick any $t_{1} \in[0,1)$ and $t_{2} \in(0,1]$ such that $t_{2}>t_{1}$. From (A.2),

$$
\mathbf{M}_{g}\left(\mathrm{t}_{1}\right)=\overline{\mathbf{M}}_{g}\left(\overline{\mathrm{t}}_{1}\right), \quad \mathbf{M}_{g}\left(\mathrm{t}_{2}\right)=\overline{\mathbf{M}}_{g}\left(\overline{\mathrm{t}}_{2}\right)
$$

where $\bar{t}_{1}=1-t_{1}, \bar{t}_{2}=1-t_{2}$. Taylor's theorem yields

$$
\begin{aligned}
& \mathbf{M}_{g}\left(\mathrm{t}_{2}\right)=\mathbf{M}_{g}\left(\mathrm{t}_{1}\right)+\dot{\mathbf{M}}_{g}\left(\mathrm{t}_{1}\right)\left\{\mathrm{t}_{2}-\mathrm{t}_{1}\right\}+\mathrm{o}\left\{\mathrm{t}_{2}-\mathrm{t}_{1}\right\} \\
& \overline{\mathbf{M}}_{g}\left(\overline{\mathrm{t}}_{1}\right)=\overline{\mathbf{M}}_{g}\left(\overline{\mathrm{t}}_{2}\right)+\dot{\mathbf{M}}_{g}\left(\overline{\mathrm{t}}_{2}\right)\left\{\overline{\mathrm{t}}_{1}-\overline{\mathrm{t}}_{2}\right\}+\mathrm{o}\left\{\overline{\mathrm{t}}_{1}-\overline{\mathrm{t}}_{2}\right\} .
\end{aligned}
$$

With (A.3), (A.5) becomes

$$
\mathbf{M}_{g}\left(\mathrm{t}_{1}\right)=\mathbf{M}_{g}\left(\mathrm{t}_{2}\right)+\dot{\mathbf{M}}_{g}\left(\overline{\mathrm{t}}_{2}\right)\left\{\overline{\mathrm{t}}_{1}-\overline{\mathrm{t}}_{2}\right\}+\mathrm{o}\left\{\overline{\mathrm{t}}_{1}-\overline{\mathrm{t}}_{2}\right\}
$$

Substitution of (A.4) for $\mathbf{M}_{g}\left(\mathrm{t}_{2}\right)$ in (A.6) leads to

$$
0=\dot{\mathbf{M}}_{g}\left(\mathrm{t}_{1}\right)\left\{\mathrm{t}_{2}-\mathrm{t}_{1}\right\}+\mathrm{o}\left\{\mathrm{t}_{2}-\mathrm{t}_{1}\right\}+\dot{\bar{M}}_{g}\left(\overline{\mathrm{t}}_{2}\right)\left\{\overline{\mathrm{t}}_{1}-\overline{\mathrm{t}}_{2}\right\}+\mathrm{o}\left\{\overline{\mathrm{t}}_{1}-\overline{\mathrm{t}}_{2}\right\}
$$


Taking the limit as $\mathrm{t}_{2} \rightarrow \mathrm{t}_{1}$, and noting that $\bar{t}_{1} \rightarrow \bar{t}_{2}$, we obtain

$$
0=\dot{\mathbf{M}}_{g}\left(\mathrm{t}_{1}\right)+\dot{\overline{\mathbf{M}}}_{g}\left(\overline{\mathrm{t}}_{1}\right)
$$

Finally, pick $t_{2}=1$ and any $t_{1} \in[0,1)$. Repeating the above procedure and taking the limit as $t_{1} \rightarrow t_{2}$ establishes the result (A.8) for $t_{1}=1$.

Thus, since $t_{1}$ is arbitrary in (A.8), we have

$$
\dot{\overline{\mathbf{M}}}_{g}(\overline{\mathrm{t}})=-\dot{\mathbf{M}}_{g}(\mathrm{t}) \text { for all } \mathrm{t} \in[0,1]
$$

and the proof is complete. 


\section{References}

1. Lai, W.M., Hou, J.S., and Mow, V.C., A triphasic theory for the swelling and deformation behavior of articular cartilage. Journal of Biomechanical Engineering 113 (1991) 245-258.

2. Woo, S.L., Akeson, W.H., and Jemmott, G. F., Measurements of nonhomogeneous directional mechanical properties of articular cartilage in tension. Journal of Biomechanics 9 (1976) 785-791.

3. Mow, V.C. and Ratcliffe, A., Structure and function of articular cartilage and meniscus, in Basic Orthopaedic Biomechanics. Raven Press, New York (1997).

4. Sandy, J.D., Adams, M.E., Billingham, M.E., Plaas, A., and Muir, H., In vivo and in vitro stimulation of chondrocyte biosynthetic activity in early experimental osteoarthritis. Arthritis and Rheumatism 27 (1984) 388-397.

5. Setton, L.A., Mow, V.C., and Howell, D.S., Mechanical behavior of articular cartilage in shear is altered by transection of the anterior cruciate ligament. Journal of Orthopaedic Research 13 (1995) 473-482.

6. Kiviranta, I., Tammi, M., Jurvelin, J., Arokoski, J., Säämänen, A.M., and Helminen, H.J., Articular cartilage thickness and glycosaminoglycan distribution in the young canine knee joint after remobilization of the immobilized limb. Journal of Orthopaedic Research 12 (1994) 161-167.

7. Palmoski, M., Perricone, E., and Brandt, K.D., Development and reversal of a proteoglycan aggregation defect in normal canine knee cartilage after immobilization. Arthritis and Rheumatism 22 (1979) 508-517.

8. Hall, A.C., Urban, J.P., and Gehl, K.A., The effects of hydrostatic pressure on matrix synthesis in articular cartilage. Journal of Orthopaedic Research 9 (1991) 1-10.

9. Sah, R.L., Kim, Y.J., Doong, J.Y., Grodzinsky, A.J., Plaas, A.H., and Sandy, J.D., Biosynthetic response of cartilage explants to dynamic compression Journal of Orthopaedic Research 7 (1989) 619-636. 
10. Palmoski, M.J. and Brandt, K.D., Effects of static and cyclic compressive loading on articular cartilage plugs in vitro. Arthritis and Rheumatism 27 (1984) 675-681.

11. Smith, R.L., et al., Effects of fluid-induced shear on articular chondrocyte morphology and metabolism in vitro. Journal of Orthopaedic Research 13 (1995) 824-831.

12. Klisch, S.M., Van Dyke, T., and Hoger, A., A theory of volumetric growth for compressible elastic materials. Mathematics and Mechanics of Solids 6 (2001) 551-575.

13. Rodriguez, E.K., Hoger, A., and McCulloch, A.D., Stress-dependent finite growth in soft elastic tissues. Journal of Biomechanics 27 (1994) 455-467.

14. Chen, Y. and Hoger, A., Constitutive function of elastic materials in finite growth and deformation. Journal of Elasticity 59 (2000) 175-193.

15. Skalak, R., Zargaryan, S., Jain, R.K., Netti, P.A., and Hoger, A., Compatibility and the genesis of residual stress by volumetric growth. Journal of Mathematical Biology 34, (1996) 889-914.

16. Lin, I.E. and Taber, L., A model for stress-induced growth in the developing heart. Journal of Biomechanical Engineering 117, (1995) 343-349.

17. Taber, L.A. and Eggers, D.W., Theoretical study of stress-modulated growth in the aorta. Journal of Theoretical Biology 180, (1996) 343-357.

18. Taber, L., A model for aortic growth based on fluid shear and fiber stresses. Journal of Biomechanical Engineering 120, (1998) 348-354.

19. Van Dyke, T. and Hoger, A., Should the growth law be defined on the initial or the current configuration? Journal of Theoretical Biology (in review).

20. Ateshian, G.A., Warden, W.H., Kim, J.J., Grelsamer, R.P., and Mow, V.C., Finite deformation biphasic material properties of bovine articular cartilage from confined compression experiments. Journal of Biomechanics 30 (1997) 1157-1164.

21. Klisch, S.M. and Lotz, J.C., A special theory of biphasic mixtures and experimental results for human annulus fibrosus tested in confined compression. Journal of Biomechanical Engineering 122 (2000) 180-188.

22. Truesdell, C. and Toupin, R.A., The classical field theories, in Handbuch der Physik, Flügge, S., Ed., Springer-Verlag, Berlin III/1 (1960). 
23. Green, A.E. and Naghdi, P.M., On basic equations for mixtures, Quarterly Journal of Mechanics and Applied Mathematics 22 (1969) 427-438.

24. Craine, R.E., Green, A.E., and Naghdi, P.M., A mixture of viscous elastic materials with different constituent temperatures, Quarterly Journal of Mechanics and Applied Mathematics 23 (1970) 171-184.

25. Bowen, R.M., Incompressible porous media models by use of the theory of mixtures, International Journal of Engineering Science 18 (1980) 1129-1148.

26. Müller, I., A thermodynamic theory of mixtures of fluids, Archive for Rational Mechanics and Analysis 28 (1968) 1-39.

27. Krishnaswamy, S. and Batra, R., A thermomechanical theory of solid-fluid mixtures, Mathematics and Mechanics of Solids 2 (1997) 143-151.

28. Day, W.A., The Thermodynamics of Simple Materials with Memory. Springer, BerlinHeidelberg-New York (1972).

29. Rivlin, R.S., Comments on some recent researches in thermomechanics, Recent Advances in Engineering Science 8 (1973) 1-23.

30. Rivlin, R.S., Reflections of certain aspects of thermomechanics, in Contributi del Centro Linceo Interdisiplinare di Scienze Matematiche e loro Applicazioni, No. 76- Meeting on: Finite thermoelasticity. Rome 30 May - 1 June, (1986) 11-43.

31. Casey, J. and Krishnaswamy, S., A characterization of internally constrained thermoelastic materials, Mathematics and Mechanics of Solids 3 (1998) 71-89.

32. Klisch, S.M., A mixture of elastic materials with different constituent temperatures and internal constraints, International Journal of Engineering Science 40 (2001) 805-828.

33. Epstein, M. and Maugin, G.A., Thermomechanics of volumetric growth in uniform bodies. International Journal of Plasticity 16 (2000) 951-978.

34. Ericksen, J.L., Introduction to the Thermodynamics of Solids. Chapman \& Hall, London (1991).

35. Atkin, R.J. and Craine, R.E., Continuum theories of mixtures: applications. Journal of the Institute of Mathematics and its Applications. 17 (1976) 153-207. 
36. Buschmann, M.D., Kim, Y.J., Wong, M., Frank, E., Hunziker, E.B. and Grodzinsky, A.J., Stimulation of aggrecan synthesis in cartilage explants by cyclic loading is localized to regions of high interstitial fluid flow. Archive of Biochemistry and Biophysics 366 (1999) 1-7.

37. Pottenger, L.A., Webb, J.E. and Lyon, N.B., Kinetics of extraction of proteoglycans from human cartilage. Arthritis and Rheumatism 28 (1985) 323-330.

38. Sajdera, S.W. and Hascall, V.C., Proteinpolysaccharide complex from bovine nasal cartilage. A Comparison of low and high shear extraction procedures. Journal of Biological Chemistry 244 (1969) 77-87.

39. Furoto, D.K. and Miller, E.J., Isolation and characterization of collagens and procollagens. Methods in Enzymology 144 (1987) 41-139.

40. Mills, N., Incompressible mixtures of Newtonian fluids. International Journal of Engineering Science 4 (1966) 97-112.

41. Mow, V.C., Kuei, S. C., Lai, W.M., and Armstrong, C.G., Biphasic creep and stress relaxation of articular cartilage in compression: theory and experiment. Journal of Biomechanical Engineering 102 (1980) 73-84.

42. Bachrach, N.B., Mow, V.C., and Guilak, R., Incompressibility of the solid matrix of articular cartilage under high hydrostatic pressures, Journal of Biomechanics 31 (1998) 445-451.

43. Adkins, J.E., Dynamic properties of resilient materials: constitutive equations, Philosophical Transactions of the Royal Society of London. 250A (1958) 519-541.

44. Truesdell, C. and Noll, W., The non-linear field theories of mechanics, in Handbuch der physik, Berlin, Springer-Verlag III/3 (1965). 


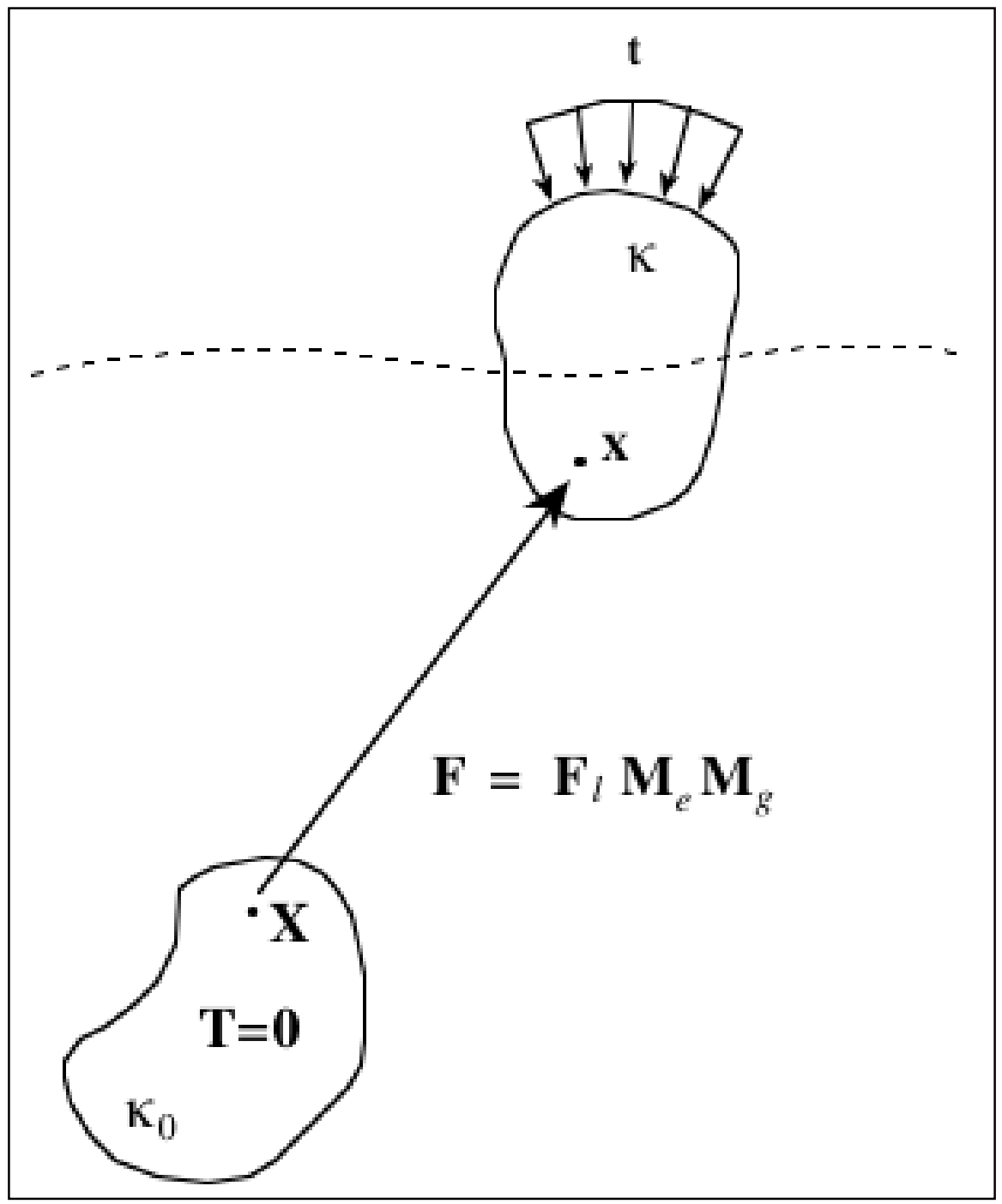

Figure 1: Schematic of the decomposition of the deformation gradient tensor $\mathbf{F}$ during growth of a thermoelastic material. A deformation gradient $\mathbf{M}_{e} \mathbf{M}_{g}$ describes growth relative to the arbitrary, fixed reference configuration $\kappa_{0}(B)$ with zero stress. An additional elastic deformation gradient due to applied loading (t) is represented by $\mathbf{F}_{l}$. 


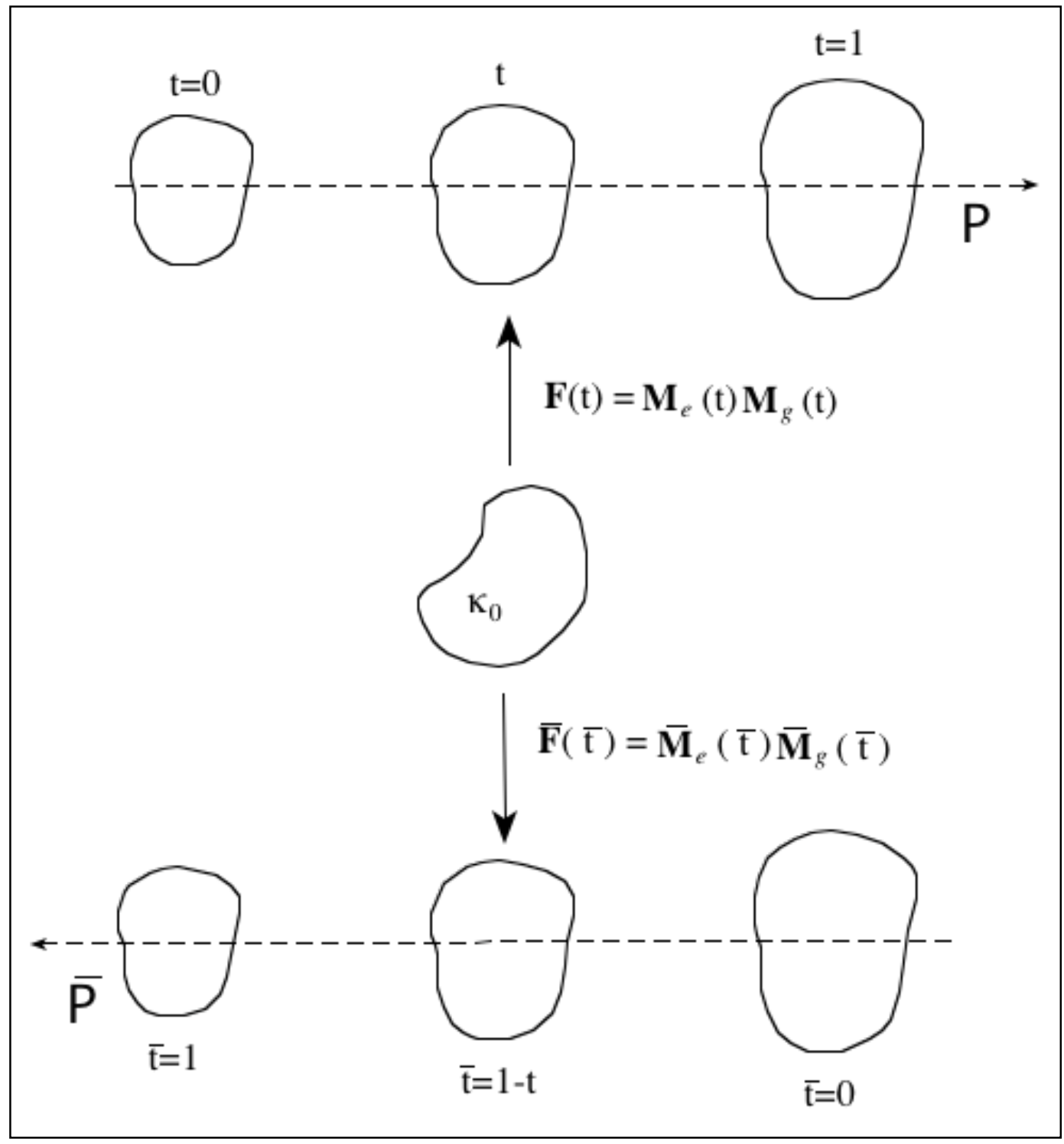

Figure 2: Schematic of the forward process $P$ and the reverse process $\bar{P}$ through the deformation space $\left(\mathbf{M}_{e}, \mathbf{M}_{g}\right)$. Relative to a fixed reference configuration $\kappa_{0}(B)$, the deformation gradient for each point along $P$ is $\mathbf{F}(\mathrm{t})=\mathbf{M}_{e}(\mathrm{t}) \mathbf{M}_{g}(\mathrm{t}), \mathrm{t} \in[0,1]$ and the deformation gradient for each point along $\bar{P}$ is $\overline{\mathbf{F}}(\mathrm{t})=\overline{\mathbf{M}}_{e}(\overline{\mathrm{t}}) \overline{\mathbf{M}}_{g}(\overline{\mathrm{t}}), \overline{\mathrm{t}} \in[0,1]$. 